\title{
MAPK pathway activation in pilocytic astrocytoma
}

\author{
David T. W. Jones · Jan Gronych $\cdot$ Peter Lichter • \\ Olaf Witt $\cdot$ Stefan M. Pfister
}

Received: 11 October 2011/Revised: 22 November 2011/Accepted: 24 November 2011/Published online: 13 December 2011

(C) The Author(s) 2011. This article is published with open access at Springerlink.com

\begin{abstract}
Pilocytic astrocytoma (PA) is the most common tumor of the pediatric central nervous system (CNS). A body of research over recent years has demonstrated a key role for mitogen-activated protein kinase (MAPK) pathway signaling in the development and behavior of PAs. Several mechanisms lead to activation of this pathway in PA, mostly in a mutually exclusive manner, with constitutive BRAF kinase activation subsequent to gene fusion being the most frequent. The high specificity of this fusion to PA when compared with other CNS tumors has diagnostic utility. In addition, the frequency of alteration of this key pathway provides an opportunity for molecularly targeted therapy in this tumor. Here, we review the current knowledge on mechanisms of MAPK activation in PA and some of the downstream consequences of this activation, which are now starting to be elucidated both in vitro and in vivo, as well as clinical considerations and possible future directions.
\end{abstract}

D.T.W. Jones and J. Gronych: These authors contributed equally.

D. T. W. Jones · J. Gronych · P. Lichter · S. M. Pfister $(\bowtie)$

Division of Molecular Genetics, German Cancer Research

Center (DKFZ), Im Neuenheimer Feld 280,

69120 Heidelberg, Germany

e-mail: s.pfister@dkfz-heidelberg.de

O. Witt · S. M. Pfister

Department of Pediatric Oncology, Hematology

and Immunology, University Hospital Heidelberg,

Im Neuenheimer Feld 430, 69120 Heidelberg, Germany

O. Witt

Clinical Cooperation Unit Pediatric Oncology, DKFZ,

Im Neuenheimer Feld 280, 69120 Heidelberg, Germany
Keywords Pilocytic - Astrocytoma - Low grade glioma . LGG $\cdot$ BRAF $\cdot$ Fusion · MAPK $\cdot$ Senescence

$\begin{array}{ll}\text { Abbreviations } \\ \text { CNS } & \begin{array}{l}\text { Central nervous system } \\ \text { Mitogen-activated protein kinase/ } \\ \text { extracellular signal-regulated kinase }\end{array} \\ \text { NF1 } & \text { Neurofibromatosis Type 1 } \\ \text { NS } & \text { Noonan syndrome } \\ \text { OIS } & \text { Oncogene-induced senescence } \\ \text { OPG } & \begin{array}{l}\text { Optic pathway glioma } \\ \text { pilocytic astrocytoma }\end{array} \\ \text { PA } & \text { Senescence-associated heterochromatin } \\ \text { SAHF } & \text { foci } \\ \text { UTR } & \text { Untranslated region }\end{array}$

Introduction

Brain tumors are the most common solid tumors of childhood, representing approximately a quarter of all pediatric neoplasia [1]. The most common histological entity in this setting is pilocytic astrocytoma (PA), which accounts for approximately $20 \%$ of brain tumors under the age of $20[2,3]$. The most frequent sites of PA occurrence are the cerebellum and the hypothalamic/chiasmatic region, but they can also arise throughout the intracranial space, including the cerebral hemispheres and brain stem, and also rarely the spinal cord [4]. They are typically seen to be slow-growing, well-circumscribed tumors, which do not invade surrounding tissues and virtually never progress to higher malignancy grades. Dissemination into the spinal canal at diagnosis has been reported, but this is a rare event occurring in only $2-3 \%$ of cases [5]. As such, 
they are classified as malignancy grade I by the World Health Organisation, and prognosis in terms of overall survival is very good: $>90 \%$ of patients survive beyond 10 years, and the majority of these long-term survivors are cured of their tumor [6,7]. Despite this, local recurrence of tumor growth, even after complete resection (as assessed by surgical report and/or postoperative MRI), occurs in about 10-20\% of cases. Rates of progression in cases where the primary lesion was not amenable to gross total resection can be as high as $50-80 \%$. Both the primary tumor and subsequent recurrence, as well as the treatments thereof, can also cause significant physical morbidity or psychosocial dysfunction [8]. The introduction of novel, targeted therapeutics could therefore be of significant benefit in treating this tumor of the childhood brain, especially since, in contrast to most other tumor entities, it can become in effect a chronic disease which might require long-term and/or repeated cycles of adjuvant therapy.

Histologically, diagnosis of PA can often be challenging. Classic presentation includes a biphasic architecture, with areas of densely packed, fibrillary tissue interspersed with looser microcystic compartments. Tumor cells usually display an elongated morphology with hair-like (piloid) tendrils that give the tumor its name. Rosenthal fibres (strongly eosinophilic structures of unknown composition) and granular bodies are also frequently observed, but are neither necessary nor sufficient for diagnosis. It is now well recognized that PAs can show widely varying morphology, with regions reminiscent of higher-grade astrocytoma, oligodendroglioma and ependymoma. Areas of necrosis and marked vascular proliferation, more often seen in highly malignant glioblastomas, are also occasionally observed [6], highlighting the clinical importance of sensitive and specific diagnostic markers for PA.

Until recently, very little was known about the genetic alterations underlying this disease. Most early copy-number studies showed either balanced karyotypes or wholechromosomal changes, with analyses of candidate genes altered in higher-grade astrocytoma (such as PTEN or TP53) revealing very few mutations [9-12]. However, the past few years have brought a substantial increase in our understanding of some of the key genetic alterations behind the development of PA, with several mechanisms converging on abnormal activation of the mitogen-activated protein kinase/extracellular signal-regulated kinase (MAPK/ERK) signaling pathway. Expanding on previous reviews in this area $[13,14]$, the present review focuses on these recent advances in our knowledge of PA tumorigenesis, and also looks ahead to how these insights might be expanded on in the future, both in terms of basic biology and with regards to transferring these data to the bedside.

\section{MAPK signaling in normal brain and high-grade astrocytomas}

Under non-pathological conditions, MAPK/ERK signaling components are expressed in most regions of the brain, and show a largely overlapping expression pattern (with the exception of MEK2, which is almost completely absent) [15]. Functionally, this signaling pathway has been implicated in various neurological processes like memory formation and pain perception (reviewed in $[16,17])$ but also in the induction of cortical neurogenesis [18] and development of the midbrain and cerebellum [19]. The latter aspects are of particular relevance with respect to PA pathogenesis, taking into account the high childhood prevalence and cerebellar preponderance of these tumors, and the accumulating evidence that neural stem/precursor cells rather than post-mitotic glial cells constitute the origin of glial neoplasms in general [20]. Indeed, there are several reports in the literature indicating that PAs express a number of markers, such as the PDGF $\alpha$ receptor, the NG2 proteoglycan, Sox10, and Olig2, similar to those of oligodendrocyte precursor cells [21-29]. However, it is also clear that the cellular effects of MAPK/ERK activation are strongly context dependent. While several studies using loss of function strategies of MEK and ERK have shown that MAPK/ERK activation promotes a neuronal fate of these early progenitors and represses glial differentiation $[18,30]$, other groups report a role for ERK activation in peri-lesional astrogliosis [31] and in oligodendrocyte differentiation in the developing mouse cortex [32]. Further work in this area is needed to investigate developmental cell-type specific effects of MAPK activation, and a possible link to a cell of origin for PA. Besides these diverse functions in the normal brain, altered MAPK/ERK signaling has also been known for some time to play a major role in the biology of higher grade astrocytomas (reviewed in $[33,34])$. The underlying aberrations, however, are different in most of these entities when compared with the common mechanisms in pilocytic astrocytoma outlined below. Rather than point mutations or gene fusions of RAF family members, these tumors often harbor high-level amplifications of upstream receptor tyrosine kinases such as EGFR and PDGFR [35, 36] or somatic mutations of the NF1 gene [36] as mechanisms for constitutive MAPK/ERK activation.

\section{Pilocytic astrocytoma and neurofibromatosis type 1}

In contrast to the somatic mutations of NF1 seen in $\sim 15 \%$ of glioblastomas [36, 37], the initial indication that MAPK signaling might play a role in the development of PAs came from clinical observations in patients with 
Neurofibromatosis Type 1 (NF1), which is caused by germline NF1 mutation. Affecting around 1 in 4,000 individuals, it is one of the more common genetic disorders and is inherited in an autosomal-dominant fashion with almost $100 \%$ penetrance, although roughly $30-50 \%$ of cases are due to new mutations $[38,39]$. The product of the NF1 gene is called neurofibromin, or NF1, and is a large (220-250 kDa) protein that acts as a GTPase-activating protein (GAP) for Ras. Loss of neurofibromin activity leads to an increase in the active form of Ras, thereby contributing to tumor formation [40]. Neurofibromin has also been implicated in maintaining progenitor cell pools in the CNS: mutations in NFl lead to an excessive accumulation of socalled O-2A precursor cells (which can give rise to oligodendrocytes or type-2 astrocytes in vitro depending on the culture model) in transgenic mice, and also result in disruption of oligodendrocyte precursor cells in zebrafish [4143]. Furthermore, loss of NF1 can also lead to mTOR/AKT pathway activation, which has been implicated in a more aggressive subset of PAs [44]. The precise role of this pathway in PAs, however, is yet to be fully determined.

NF1 is associated with an increased risk of glioma formation, and PA is one of the most commonly involved entities, accounting for about half of all NF1-associated gliomas $[45,46]$. Roughly $15 \%$ of NF1 patients have PAs, particularly in the optic pathway [47], and optic pathway gliomas are considered one of the diagnostic criteria for the syndrome [48]. Conversely, about a third of tumors in the optic pathway are PAs [49] and roughly $10 \%$ of all PAs are NF1-associated, suggesting that PA patients, particularly with optic pathway tumors, should be examined for clinical signs of NF1 [50]. Mutation screening of NF1 can be difficult, since the gene comprises 58 exons spread over nearly $300 \mathrm{~kb}$ of chromosome 17 and the types of mutations observed can be complex. With the application of high-throughput sequencing techniques, however, it will be interesting to see whether either somatic NF1 mutations or clinically undiagnosed germline NFl alterations are also seen in PAs.

One further important question that remains to be answered, with respect to the role of $N F 1$ in PA, is whether it is possible to predict which patients with a clinical diagnosis of neurofibromatosis type I will go on to develop a pilocytic astrocytoma (or other glioma), and which of those patients will suffer most from, for example, vision loss or impairment. It was recently shown that there appears to be some degree of genotype-phenotype correlation in this syndrome, with NF1 patients harboring a mutation in the first third of the gene more likely to develop an optic pathway glioma [51]. Whether these findings can be further expanded on, in order to clarify a link between specific NFl alterations and risk of developing a PA, will be of particular interest both biologically and from a clinical perspective.

In addition to NF1, pilocytic astrocytoma has also been reported to occur in a small number of patients with Noonan syndrome (NS) [52-54]. As with many of the neuro-cardio-facial-cutaneous syndromes, NS is characterized by germline alterations in MAPK pathway genes, particularly PTPN11, SOS1, and KRAS (reviewed in [55]). Whether this link is more than coincidental, and whether PA is observed in any other hereditary MAPK pathway disorders, are yet to be determined.

\section{Gene fusions involving $B R A F$ are a defining feature of pilocytic astrocytoma}

While a familial tumor syndrome first provided indirect evidence for a link between MAPK signaling and PAs, truly compelling evidence for the fundamental role of this pathway in PA tumorigenesis came with the finding of a highly frequent somatic rearrangement occurring in the majority of sporadic cases. Focal duplication of approximately $2.5 \mathrm{Mb}$ at 7q34 was reported as being a strikingly common feature in pilocytic astrocytoma in 2008 [56-58], although the exact significance of this alteration was not immediately clear. Shortly thereafter, however, it was shown that a gain in this region resulted in a novel fusion between KIAA1549 (a large, as-yet uncharacterized gene) and the $B R A F$ oncogene $[59,60]$. The study by Jones et al. [59] further demonstrated that this fusion resulted in constitutive activation of BRAF kinase activity, and was able to transform NIH-3T3 cells. Since then, several additional studies have reported similar findings, and contributed to expanding our understanding of the frequency and specificity of this alteration [61-69]. The frequency of this change stated in the literature varies from 50 to $100 \%$ depending on the demographics of the patients investigated, and a total of five different exonic combinations of the two genes have been described (see Fig 1). The most common (KIAA1549 exons 1-16 and BRAF exons 9-18, or $\mathrm{K}: \mathrm{B}^{16 \_9}$ ) comprises roughly $60 \%$ of fusion events, with $\mathrm{K}: \mathrm{B}^{15 \_9}$ accounting for $\sim 30 \%$ and $\mathrm{K}: \mathrm{B}^{16 \_11} \sim 10 \%$, with minor contributions from rare variants. In all cases, however, the fusion leads to loss of the BRAF N-terminal autoregulatory domain and subsequent activation of the kinase domain. This is in keeping with BRAF fusions previously seen in a small fraction of thyroid tumors and large congenital melanocytic nevi, as well as rare RAF fusions recently identified in melanoma, and in prostate and gastric tumors [70-72]. Studies on larger numbers of PA cases are now starting to identify links between clinical parameters and BRAF fusion (as discussed below), but this is still an 


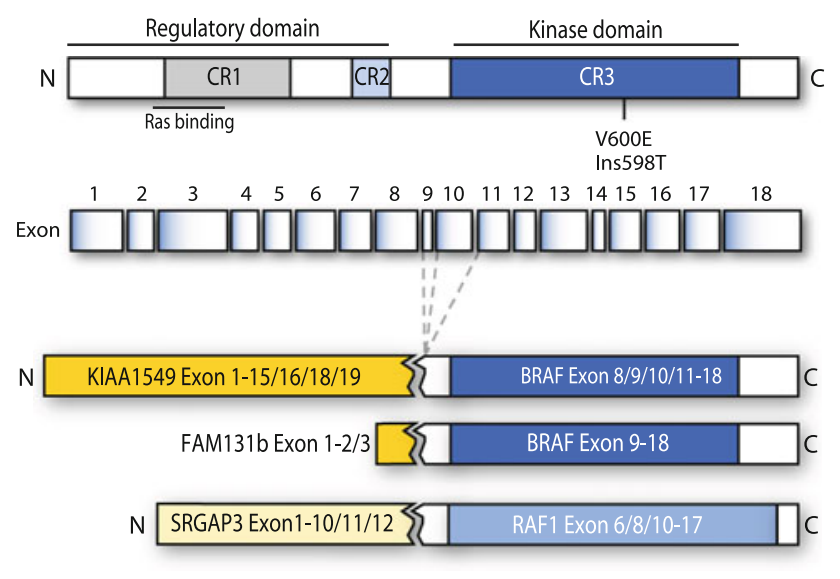

Fig. 1 Schematic representation of the genomic and protein structure of human BRAF and the fusion products detected in pilocytic astrocytoma. The gene fusions with their indicated fusion partners and break points in all cases result in a loss of the amino-terminal autoregulatory domain. This, as well as the V600E point mutation and the Ins598T insertion in the full length protein, results in constitutive activity of the kinase domain independent of upstream Ras status. CR1-3 conserved region 1-3

area which will greatly benefit from further (prospective) investigation in larger cohorts.

Apart from its high frequency, another striking feature of the KIAA1549:BRAF fusion is its exquisite specificity to PAs. Several reports looking at various additional low and high malignancy grade pediatric brain tumors have found no evidence for the fusion gene in these additional entities $[65,67,68,73]$. Whilst some studies have observed a small number of cases of grade II astrocytoma, mixed oligoastrocytic tumors, or pilomyxoid astrocytomas harboring the fusion gene, it is not currently clear whether these might in fact represent misdiagnosed PAs with an unusual histological composition [59, 60, 62, 64]. In the case of pilomyxoid astrocytoma, in particular, it seems that this may be part of a spectrum of PA morphology, perhaps representing a slightly earlier, less-differentiated stage in the tumor's development, rather than an entirely distinct entity. Indeed, there are reports of primary pilomyxoid astrocytomas diagnosed early in life recurring later as prototypic pilocytic tumors [74]. It is also not yet clear whether the worse prognosis initially ascribed to these tumors (warranting a malignancy grade II classification by the WHO) is independent of the fact that they occur predominantly in surgically less-accessible regions and in younger patients.

It is thus tempting to speculate that there may be something unique to the PA cell of origin that allows this fusion to drive oncogenesis solely in this tumor type. Perhaps there is a 'Goldilocks' cell for this fusion, such that the signaling induced is 'just right' to be tolerated and induce transformation, where other cells may be insensitive to the stimulus, die, or undergo immediate growth arrest. The multiple, cell type-specific roles for MAPK signaling in the brain, as discussed above, further support the hypothesis that only a distinct cell type with certain inherent properties and interactions with the microenvironment might be vulnerable to this mechanism of BRAFmediated transformation. An additional possibility is that the transcriptional program of the PA precursor may lead to a particular configuration of active and inactive chromatin within the nucleus that brings the two genes in close proximity and potentiates recombination. Elucidation of the exact processes through which the $7 \mathrm{q} 34$ duplication arises may help to shed light on the question of its specificity. Whilst the mechanism is still not entirely clear, a recent study looking at the mapping of genomic breakpoints suggested a possible recombination mechanism. Lawson et al. [75, 76] identified an enrichment of sequence microhomology, complex rearrangements, and proximity to repeat elements, suggesting that the process of microhomology-mediated break-induced replication (MMBIR) may be involved.

The presence of microhomology, 'filler' DNA and sometimes complex rearrangements was also noted by Cin et al. [61], who further reported a second mechanism of BRAF fusion in a small number of PAs. In three cases identified to date, a $\sim 2.5-\mathrm{Mb}$ deletion at $7 \mathrm{q} 34$, telomeric to $B R A F$, results in a fusion between it and the uncharacterized gene $F A M 131 B$ (Fig. 1). The resulting protein again retained only the kinase domain of BRAF, and functional analysis demonstrated constitutive kinase activity as well as transformation of NIH-3T3 cells. Interestingly, the breakpoints identified were close to the $5^{\prime}$ end of $F A M 131 B$ and consisted primarily of $5^{\prime}$ UTR. Only a short fragment of the FAM131B protein is therefore included in these fusions, suggesting that the $5^{\prime}$ partner gene may be acting primarily to induce transcription of the fusion and provide a carrier for the BRAF kinase domain, rather than having a functional protein role.

\section{Alternative mechanisms of MAPK activation}

The second most common change seen in PAs also involves the BRAF gene, but consists of single amino acid changes rather than gene rearrangement. Most often this is the hotspot valine to glutamate change at position 600 (V600E), first identified in 2002 and since then reported in a large number of tumor types ([77]; and see the Catalogue of Somatic Mutations in Cancer (COSMIC) at http://www. sanger.ac.uk/genetics/CGP/cosmic/ for further details). This mutation has been extensively characterized and is a welldocumented oncogenic lesion [78, 79]. In addition, however, a novel 3-bp (TAC) insertion encoding an extra threonine 
residue adjacent to the $\mathrm{V} 600$ hotspot codon has also been reported in a few cases of PA [69, 80-82]. This alteration, referred to as $\mathrm{BRAF}^{\text {ins598T }}$ or simply $\mathrm{BRAF}^{\text {insT }}$, has been shown to induce constitutive kinase activity at a level similar to the V600E change, and it also shows transforming ability in vitro $[80,81]$.

In stark contrast to the KIAA1549:BRAF fusion, the V600E mutation does not appear to be specific to a brain tumor entity. Two recent studies looking at BRAF mutational status in a variety of entities, including a report from the von Deimling group on more than 1,300 CNS tumors, showed the presence of mutation in various subtypes [73, 82]. Particularly high incidence was seen in pleomorphic xanthoastrocytoma and ganglioglioma, suggesting that BRAF activation has a broader role to play in brain tumorigenesis, particularly in tumors of lower malignancy grades. The elucidation of the exact downstream pathways involved is therefore a key target for future research.

Another somatically mutated gene in PA, first reported several years prior to the discovery of $\mathrm{BRAF}^{\mathrm{V} 600 \mathrm{E}}$, is $K R A S$. In fact, one of the first identified somatic alterations in pilocytic astrocytoma was a KRas ${ }^{\mathrm{Q} 61 \mathrm{E}}$ mutation [83]. Further mutations in the hotspot codons 12, 13, and 61 have subsequently been found in several larger, independent tumor series, but only at low frequency $(<5 \%)[61,62$, $84,85]$. No mutations have yet been reported in HRAS or $N R A S$ in PA, suggesting that KRAS is likely the predominant isoform involved in the tumorigenic processes of PA. Intriguingly, there is also evidence that tumor development in an NF1 mouse model arises specifically from preferential activation of KRAS in astrocytes, further supporting this hypothesis [86].

A further uncommon, yet still recurrent, mechanism of MAPK pathway activation in pilocytic astrocytoma, so far reported in only a few cases, is fusion of a second Raf kinase family member, $R A F 1$ (or $C R A F$ ) [61, 62, 81]. As with the more frequent $B R A F$ alteration, fusion between $R A F 1$ and SRGAP3 is also mediated by a tandem duplication event, occurring at $3 \mathrm{p} 25$. Several fusion junctions have been reported, but all result in a truncated RAF1 kinase domain (Fig. 1). The fusion protein has also been shown to possess constitutive kinase activity and transforming ability [81]. Like the 7q34 duplication, this alteration appears to be highly specific to PA, and it has so far not been reported in any other tumor type.

Very few other genes have been reported to be mutated in PAs, including those which are commonly associated with higher grade astrocytomas. There are reports of individual cases with mutations in TP53 and PTEN, for example [87-90], and one report described a high frequency of TP53 mutation. However, these findings have not been replicated in more recent cohorts, and these genes are currently not thought to play a major role in the development of pilocytic astrocytoma.

The various alterations in the MAPK pathway described here are usually seen to be mutually exclusive within PA, suggesting that a single hit in the pathway may be sufficient for transformation in most cases. However, rare co-occurrence of $\mathrm{BRAF}^{\mathrm{V} 600 \mathrm{E}}$ with either KIAA1549:BRAF fusion or clinically diagnosed NF1 has also been reported [61, 64, 80]. Indeed, one patient apparently carried all three of these alterations [61]. The number of cases involved is currently too small to assess whether patients with multiple hits in the pathway generally show a worse clinical outcome.

Taken together, at least one hit in the MAPK pathway has been identified in approximately $80-90 \%$ of PA cases reported to date (see Fig. 2a). The question of which alterations are responsible for the remaining cases remains unclear, but is the subject of ongoing investigation in largescale genomics projects such as the International Cancer Genome Consortium, and elsewhere [91]. These studies should tell us in the foreseeable future whether this tumor is truly associated solely with hits in the MAPK pathway, or whether it also depends on as-yet unidentified secondary alterations.

\section{Clinicopathological correlates of MAPK alterations}

With the growing number of reports on the incidence of MAPK pathway alterations in PA, trends of association with clinico-pathological parameters are starting to emerge. One of the earliest recognized features, now confirmed in several larger series, is an association between tumor location and the types of MAPK aberration observed. Infratentorial tumors (most commonly in the cerebellum) tend to show a very high frequency of KIAA1549:BRAF fusion, while supratentorial tumors generally show a lower proportion of fusion-positive tumors, but an increased incidence of BRAF $^{\mathrm{V} 600 \mathrm{E}}$ mutation [56, 61, 63-65, 82] (Fig. 2b). The reason for this discrepancy, and its potential impact on tumor behavior, is not currently clear, but the fact that a similar propensity has been observed in multiple independent studies suggests a genuine phenomenon. No histological differences between $B R A F$ fusion and mutant tumors have been reported.

It has become apparent that there is a striking difference in the proportion of $B R A F$ fusion-positive cases between pediatric and adult cases of PA, with the frequency getting much lower with increasing age at diagnosis [63]. An influence of age on genetic alterations has also been previously reported for larger-scale changes, with wholechromosome gains (particularly chromosomes 5 and 7) being significantly more common in adult patients and almost absent in the youngest patients [9]. The difference 

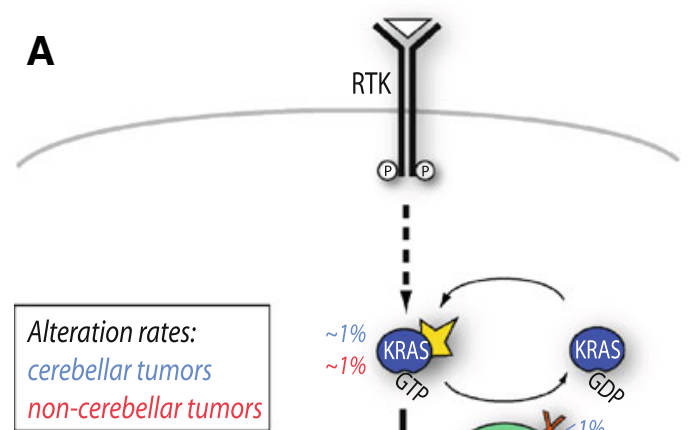

i

i

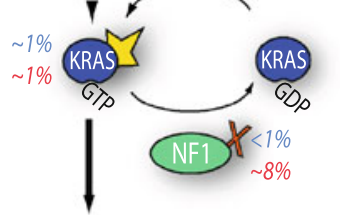

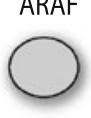

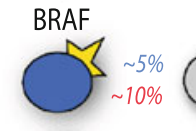

RAF1

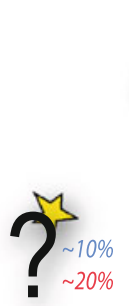

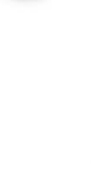
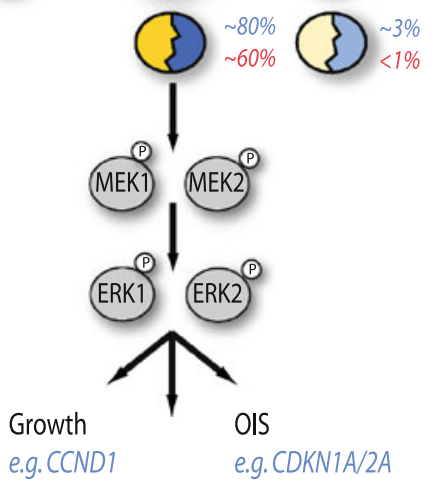

Differentiation

e.g. GFAP

Fig. 2 Distribution of oncogenic hits in the MAPK/ERK pathway by tumor location. a Representation of the MAPK/ERK signal cascade. Aberrations activating the pathway, i.e. activating mutations (star), inactivating mutation of the repressor NF1 $(X)$, fusions proteins and unknown alterations (?), are indicated with their frequencies in cerebellar and non-cerebellar PAs. Activation of this signaling pathway can induce various cellular responses like cell growth, differentiation, and oncogene-induced senescence $(O I S)$. b The

in frequency of $B R A F$ fusion is in contrast to the situation in grade II astrocytomas, where signature changes that are frequent in adult tumors (such as IDHI and TP53 mutation) are much less common in children [92]. This raises an additional consideration in respect to the diagnostic utility of BRAF fusion and IDHI or TP53 mutation in different age groups. The presence of a fusion in pediatric cases and IDH1 or TP53 mutation in adult cases gives support for a diagnosis of PA versus grade II astrocytoma, respectively. It seems, however, that the absence of either change may rather indicate a grade II astrocytoma in young patients but pilocytic astrocytoma in adults. This feature, however, will require careful assessment in larger, well-characterized series.

An additional question, which will need addressing in a larger series, is the impact of KIAA1549:BRAF and other
B

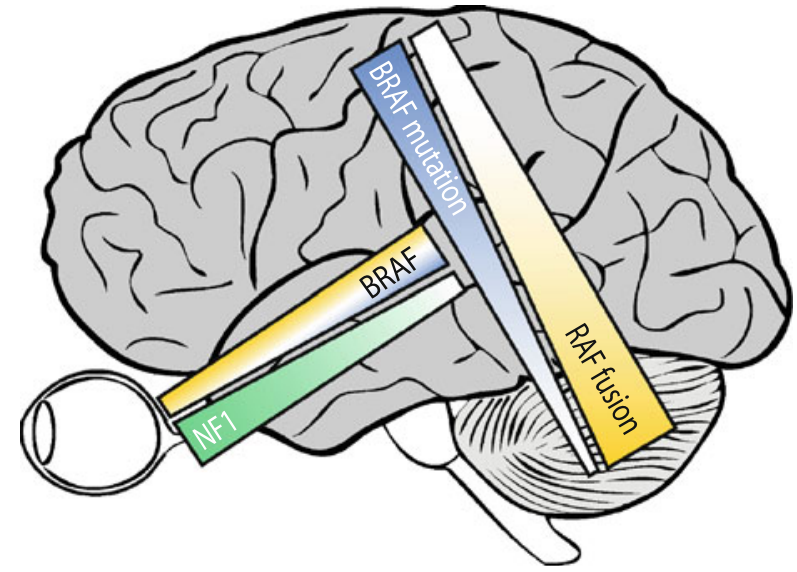

various MAPK pathway alterations are unevenly distributed in tumors of different locations. NF1 mutation is mainly seen in optic pathway gliomas but also occasionally in other locations. Tumors in other brain regions are dominated by RAF activation, with fusions occurring primarily in cerebellar tumors and mutations in supratentorial PAs. For the more infrequent hits, no prevalent locations can be given

alterations on the prognosis of patients with PA. A study by Hawkins and colleagues reported an association of $B R A F$ fusion with a favorable prognosis amongst 70 low-grade astrocytomas (PAs and grade II diffuse/pilomyxoid astrocytomas), which they deemed 'clinically relevant'; i.e., which were incompletely resected due to localization outside of the cerebellum [64]. In this subgroup, fusionpositive tumors had a hazard ratio of 0.28 (95\% CI, 0.14-0.58) for tumor progression compared with fusionnegative counterparts. In contrast, looking solely at pilocytic astrocytomas and in all tumor locations, Cin et al. [61] did not find an association of KIAA1549:BRAF fusion with progression-free survival. They did, however, report both an age of $\leq 1$ year and incomplete tumor resection as independent factors of poor prognosis upon multivariate analysis of 93 cases. 


\section{Oncogene-induced senescence in PA}

In addition to clarifying the relationship between classes of MAPK alteration and clinical/pathological factors, the next advances in our understanding of PA biology will come from determining the precise downstream consequences of MAPK pathway activation in this tumor. Two recent reports have taken steps addressing this issue, by demonstrating that MAPK activation in PA leads to oncogeneinduced senescence (OIS) [93, 94]. OIS is a process of growth arrest occurring as a tumor-suppressive mechanism in response to oncogene activation [95]. Several links have previously been made between the MAPK signaling pathway and induction of OIS. Since being first described as a response to oncogenic Ras signaling, senescence has now also been shown to be induced by BRAF activation and loss of NF1 activity [96, 97]. The papers on OIS in PAs by Jacob et al. and Raabe and colleagues [93, 94] showed that this phenomenon occurs both in vitro, in models of BRAF activation in neural precursors, and also in cells from primary tumor samples (Fig. 3). The Jacob et al. study further demonstrated a more generalized mRNA expression pattern of OIS activation in two independent primary tumor cohorts. It now seems likely that this process plays a major role in restricting PA to its relatively slow growth pattern and generally more benign behavior compared with higher grade astrocytomas.

Interestingly, both these studies also pointed to a key role of the p16 tumor suppressor in mediating the OIS process in PAs, with the study of Raabe et al. reporting a link between lack of p16 immunopositivity and worse clinical outcome.

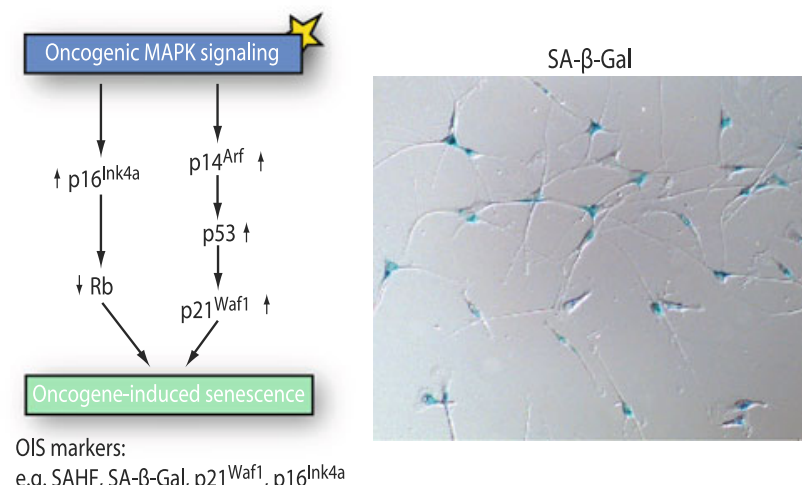

Fig. 3 Oncogene-induced senescence in pilocytic astrocytoma. Left Excessive MAPK activation can induce irreversible cell cycle arrest via the $\mathrm{p} 16^{\text {Ink } 4 \mathrm{a}} / \mathrm{Rb}$ or the $\mathrm{p} 14^{\text {Arf }} / \mathrm{p} 53$ pathway. This state can be determined, e.g., by the appearance of senescence-associated heterochromatin foci $(S A H F)$, immunostaining for $p 16^{\text {Ink4a }}$ and $p 21^{\text {Wafl }}$ or by the characteristic staining for senescence-associated- $\beta$-galactosidase $(S A-\beta-G a l)$ activity. Right Primary cultured pilocytic astrocytoma cells display clear $S A-\beta-G a l$ activity (image kindly provided by Dr. Karine Jacob, McGill University Health Center Research Institute)
This link fits with the observation of p16 loss in a subset of PAs with histologically anaplastic features and poorer prognosis [44]. Further investigation is needed to fully assess the contribution of p16 immunostaining for prognostication with respect to PA behavior. In addition, the growth repression due to OIS in PAs appears to be less than that in melanocytic nevi, which also frequently exhibit BRAFV600E-dependent OIS and thereby an early growth arrest [97], since PAs can often grow to quite a considerable size before presenting as a symptomatic tumor. Hence, it will be of great interest for the understanding of PA biology to further investigate the mechanisms regulating the balance between growth and senescence in the context of particular mitogenic stimuli in the PA cell of origin. This may also prove to have relevance for informing treatment decisions, for example when to give adjuvant therapy versus a 'watch and wait' approach.

\section{Mouse models of MAPK-driven gliomagenesis}

Activation of MAPK/ERK signaling in the murine brain has repeatedly been used for the generation of experimental gliomas. Various approaches initially utilized overexpression of oncogenic Ras, which alone or in combination with PI3K pathway activation or loss of the tumor suppressors Ink4a/Arf, p53 or PTEN, resulted in the development of grade II-IV glioma [98-104]. The first model of PA mimicked the optic pathway tumors resulting from germline NF1 alteration. Constitutive homozygous deletion of NF1 is embryonically lethal in mice [105], while heterozygous animals do not develop astrocytic tumors [106]. The group of David Gutmann therefore applied an inducible knockout approach to specifically delete the residual copy of NF1 in astrocytes of otherwise NF1-heterozygous mice ( $\mathrm{NF} 1^{\text {flox/mut }}$; GFAP-Cre), a situation corresponding to that in NF1 patients [107]. These animals developed benign lesions in the optic pathway with histologic similarity to PA. Interestingly, homozygous deletion in astrocytes alone was not sufficient for tumor induction in these studies, suggesting that NF1 heterozygous cells in the microenvironment and other microenvironmental signals may contribute to the development of these optic pathway gliomas (OPGs) [108, 109]. Two recent studies propose that this effect is due to CXCL12 secretion from stromal cells and infiltrating microglia, which show a growth promoting effect on NF1-/- cells [110, 111]. In fact, conditional deletion of NF1 in astrocytes, glial precursors and neurons adjacent to the retina using GFAP-Cre has been shown to induce OPGs, although with only a low penetrance [112]. Notably, loss of p53 either alone or with PTEN deletion in addition to NF1 deficiency both result in the development of glioblastoma [113, 114]. 
The first study to investigate the gliomagenic potential of the RAF gene family was published by the group of Eric Holland in 2008. They used the RCAS/Ntv-a somatic retroviral gene transfer system to transduce nestin-positive neural progenitor cells in vivo with an N-terminally deleted, constitutively active variant of the human RAF1 gene. Expression of this RAF1 variant alone induced only hyperplastic lesions, but in conjunction with Ink4a/Arf loss or AKT overexpression it gave rise to high-grade gliomas histologically similar to tumors induced with oncogenic KRAS [115]. Robinson et al. used the same system for expression of wild-type and V600E-mutant BRAF in vivo. Again, BRAF expression resulted in the induction of tumors only when combined with AKT activation or Ink4a/ Arf knockout [116]. The same group recently published that MAPK/ERK pathway activation by the downstream effector MEK instead of BRAF is also capable of driving gliomagenesis in this setting, but the induced tumors were again of higher grades [117].

We recently extended this analysis of BRAF in the RCAS system with truncated versions of wild-type and mutant BRAF corresponding to the portion retained in the most frequent fusion genes [118]. While confirming previous results using the full length constructs, the truncated form of mutated BRAF was sufficient to induce tumorigenesis without any additional oncogenic hit. The respective tumors resembled PA not only on histological and immunohistochemical levels, with fibrous tissue texture, strong GFAP and phospho-Erk immunoreactivity as well as a low proliferation index (Fig. 4a), but also with respect to their benign behavior, since tumor-bearing animals do not typically succumb to the disease, even without treatment (authors' unpublished observations). In vitro, the oncogenic BRAF variant induced MAPK signaling and proliferation in primary astrocytes, both of which effects could be abrogated by pharmacologic BRAF inhibition [118] (Fig. 4b). These results confirmed that MAPK activation driven via BRAF is sufficient to induce PA in vivo without requiring a cooperating second alteration. It will now be of great interest to exploit this model system for further investigation of PA tumor biology and for testing novel targeted therapies in a pre-clinical setting, in order to translate these advances into a benefit for PA patients.

\section{Clinical challenges and future directions for treatment of PAs}

There are a number of clinical challenges with respect to the management of patients with pilocytic astrocytoma. Surgical resection is the treatment of choice for pilocytic astrocytoma in children, and, in comparison with highergrade gliomas and other malignant brain tumors, this usually results in excellent long-term survival rates $[7,8$, 119-121]. Because of the long-term survival of the vast majority of patients, pilocytic astrocytoma is viewed as a chronic disease by many pediatric neurooncologists. Treatment approaches should therefore aim for efficacy not only in terms of tumor growth control but also in terms of managing tumor- and treatment-related acute and long-term toxicity, and quality of life. For example, patients with supratentorial midline tumors frequently present with visual symptoms including nystagmus and loss of visual acuity. In addition, disruption of the hypothalamic region can result in endocrine problems such as growth failure, delayed onset of puberty, or pituitary gland dysfunction. Infants with supratentorial midline tumors may also suffer from diencephalic syndrome, which is associated with failure to thrive, weight loss, and cachexia. PAs located in the posterior fossa cause headache, nausea, and vomiting due to obstruction of the fourth ventricle and subsequent increased intracranial pressure, as well as ataxia due to pressure on the cerebellum. Tumors located in the cerebral hemispheres are associated with epileptic seizures or hemiplegia, whilst those arising in the brain stem can produce cranial nerve palsies including oculomotor or facial nerve palsy, swallowing difficulties, and tongue atrophy.

Although outcome after surgery is generally good, complete surgical resection can only be achieved in around half of all cases, when the tumor is located in surgically accessible sites such as the posterior fossa. If the tumor involves the optic pathway, or the hypothalamic or thalamic regions, complete removal is impossible in a majority of patients. In the case of tumor progression, nonsurgical treatment strategies including chemotherapy and radiation therapy are usually implemented. Chemotherapy protocols are frequently based on a carboplatinum/vincristine regimen, and are most often applied to younger children and patients with NF1 suffering from non-resectable progressive disease [120,122, 123]. Older children will typically receive local radiation therapy in the event of tumor progression. These additional treatments increase the risk of patients experiencing more severe side effects.

With the discovery of BRAF alterations and constitutive activation of the downstream MAPK-pathway in the majority of cases of pilocytic astrocytoma, targeted therapies are now being recognized as potential novel treatment approaches. There are currently a number of preliminary phase I/II clinical trials ongoing which are testing small molecule kinase inhibitors targeting the MAPK or related pathways, including: MEK inhibitors (ClinicalTrials.gov: NCT01386450, NCT01089101), RAF/multiple tyrosine kinase inhibitors such as Sorafenib (ClinicalTrials. gov: NCT01338857), and mTOR inhibitors in patients with and without NF1 (ClinicalTrials.gov: NCT01158651, NCT00782626). The outcome of these early clinical 
Fig. 4 BRAF-induced murine pilocytic astrocytoma. a Tumors induced by somatic gene BRAF display histologic fiber-rich tissue as well as a low proliferation index (as assessed by Ki67 immunopositivity), clear GFAP immunopositivity, and a strong activation of MAPK signaling (illustrated by ERK-phosphorylation; $p E r k$ ). b Expression of activated BRAF induces proliferation in primary murine astrocytes in vitro, which can be markedly reduced by treatment with the kinase inhibitor Sorafenib transfer of an activated form of features of human PA, including
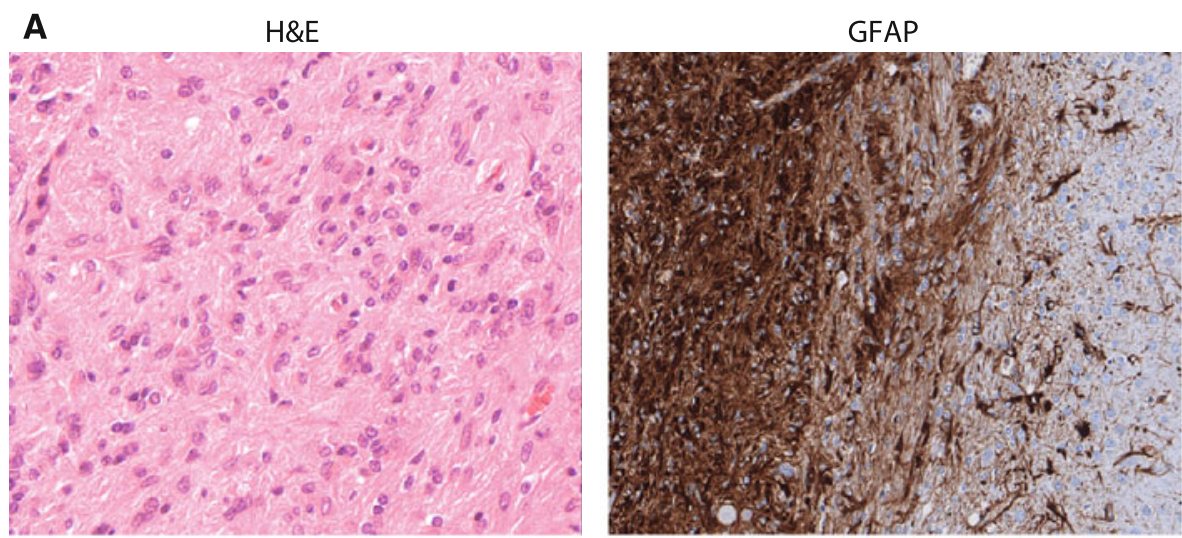

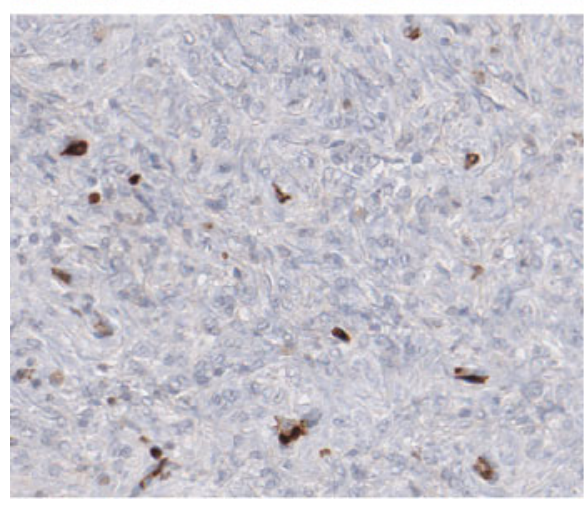

Ki67

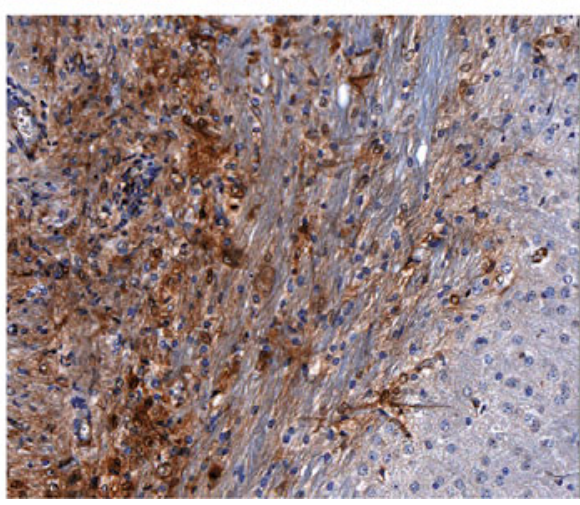

pErk

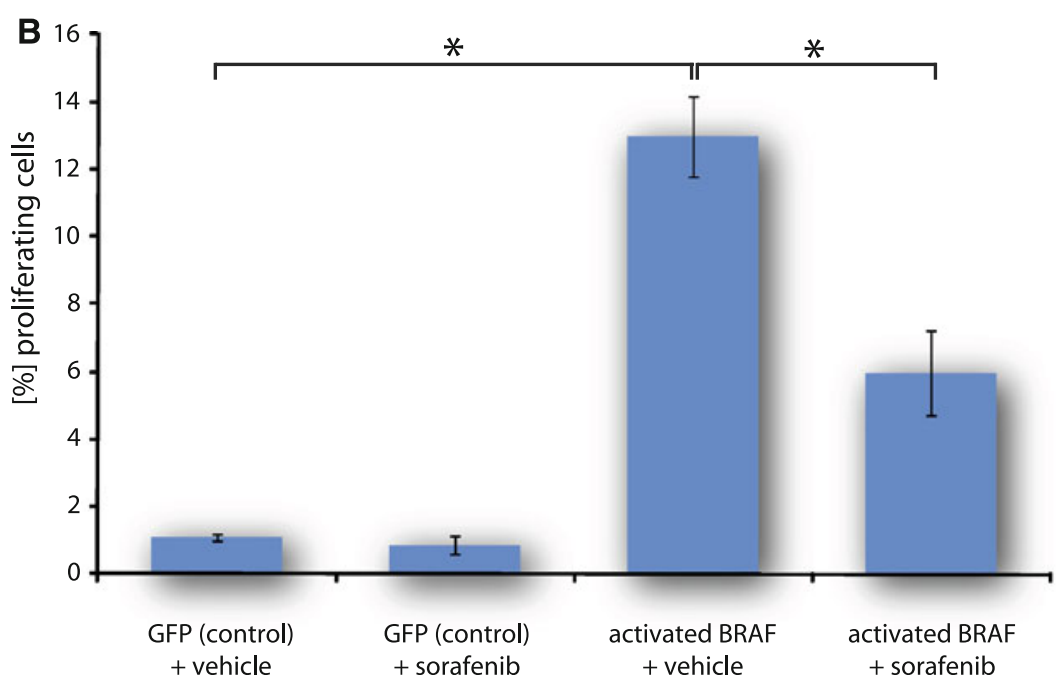

studies will be of great importance for the further development of larger clinical trials for patients with pilocytic astrocytoma in the forthcoming years.

Further advances in understanding the biology of pilocytic astrocytoma, particularly in further elucidating the precise roles and downstream effects of MAPK pathway activation, also have the potential to greatly improve diagnosis and prognostication of PAs, and also to offer additional targets for novel therapeutic strategies. For example, little is known about the underlying biological factors determining the likelihood of progression of pilocytic astrocytomas: whereas many of the patients with noncompletely resected tumors will exhibit early progression within 1-2 years after surgery, about $20 \%$ will show longterm stability over more than 10 years without any intervention. In contrast to older children, small infants below 1 year of age have a poor prognosis and even succumb to their disease in a large proportion of cases. Furthermore, the biological factors determining spinal or leptomenigneal dissemination are not known, nor are any predictors of 
response to chemotherapy or radiation treatment. All these are areas which would benefit from additional research and are currently under intensive investigation.

\section{Summary}

In conclusion, recent results in this field have proven highly significant both in terms of dramatically increasing our understanding of the basic biology behind pilocytic astrocytoma, and providing opportunities for rapid translation into clinical benefit for patients. However, there remain a number of pressing unanswered questions, including: What are the precise downstream effects of MAPK signaling activation in this tumor that lead to its behavior? Is PA a single-pathway disease, and what occurs in the remaining 10-20\% of PA cases without apparent MAPK signaling alterations? How does cerebellar PA relate to supratentorial PA or the pilomyxoid variant? And can we identify clinically relevant subgroups (such as very young patients) with inferior prognosis? We expect that currently ongoing efforts, such as large-scale whole genome sequencing within the International Cancer Genome Consortium (ICGC) Pediatric Brain Tumor Project (http://www.pedbrain.org) as well as many other studies worldwide, will be able to build on the strong foundation provided in the last few years, and drive continued progress towards combating the most common pediatric brain tumor.

Open Access This article is distributed under the terms of the Creative Commons Attribution Noncommercial License which permits any noncommercial use, distribution, and reproduction in any medium, provided the original author(s) and source are credited.

\section{References}

1. Cancer Research UK (2010) Statistical report: CancerStats childhood cancer. CRUK, London

2. Pfister S, Witt O (2009) Pediatric gliomas. Recent Results Cancer Res 171:67-81

3. Central brain tumor registry of the United States (2010) Statistical report: Primary brain and central nervous system tumors diagnosed in the United States 2004-2006. CBTRUS, Hinsdale

4. Stokland $\mathrm{T}$ et al (2010) A multivariate analysis of factors determining tumor progression in childhood low-grade glioma: a population-based cohort study (CCLG CNS9702). Neuro Oncol 12(12):1257-1268

5. von Hornstein S et al (2011) Impact of chemotherapy on disseminated low-grade glioma in children and adolescents: report from the HIT-LGG 1996 trial. Pediatr Blood Cancer 56(7):1046-1054

6. Louis DN et al (2007) WHO Classification of Tumours of the Central Nervous System. IARC Press, Lyon

7. Ohgaki H, Kleihues P (2005) Population-based studies on incidence, survival rates, and genetic alterations in astrocytic and oligodendroglial gliomas. J Neuropathol Exp Neurol 64(6): 479-489
8. Armstrong GT et al (2011) Survival and long-term health and cognitive outcomes after low-grade glioma. Neuro Oncol 13(2):223-234

9. Jones DTW et al (2006) Genomic analysis of pilocytic astrocytomas at $0.97 \mathrm{Mb}$ resolution shows an increasing tendency toward chromosomal copy number change with age. J Neuropathol Exp Neurol 65(11):1049-1058

10. Schrock E et al (1996) Recurrent gain of chromosome arm 7q in low-grade astrocytic tumors studied by comparative genomic hybridization. Genes Chromosomes Cancer 15(4):199-205

11. Walker $\mathrm{C}$ et al (2001) Characterisation of molecular alterations in microdissected archival gliomas. Acta Neuropathol 101(4): 321-333

12. White FV et al (1995) Nonrandom chromosomal gains in pilocytic astrocytomas of childhood. Hum Pathol 26(9):979-986

13. Jeuken JW, Wesseling P (2010) MAPK pathway activation through BRAF gene fusion in pilocytic astrocytomas; a novel oncogenic fusion gene with diagnostic, prognostic, and therapeutic potential. J Pathol 222(4):324-328

14. Tatevossian RG et al (2010) MAPK pathway activation and the origins of pediatric low-grade astrocytomas. J Cell Physiol 222(3):509-514

15. Di Benedetto B et al (2007) Differential mRNA distribution of components of the ERK/MAPK signalling cascade in the adult mouse brain. J Comp Neurol 500(3):542-556

16. Ji RR et al (2009) MAP kinase and pain. Brain Res Rev 60(1):135-148

17. Sweatt JD (2001) The neuronal MAP kinase cascade: a biochemical signal integration system subserving synaptic plasticity and memory. J Neurochem 76(1):1-10

18. Samuels IS et al (2008) Deletion of ERK2 mitogen-activated protein kinase identifies its key roles in cortical neurogenesis and cognitive function. J Neurosci 28(27):6983-6995

19. Sato T, Nakamura H (2004) The Fgf8 signal causes cerebellar differentiation by activating the Ras-ERK signaling pathway. Development 131(17):4275-4285

20. Liu C et al (2011) Mosaic analysis with double markers reveals tumor cell of origin in glioma. Cell 146(2):209-221

21. Bannykh SI et al (2005) Oligodendroglial-specific transcriptional factor sox10 is ubiquitously expressed in human gliomas. J Neurooncol 76(2):115-127

22. Bouvier C et al (2003) Shared oligodendrocyte lineage gene expression in gliomas and oligodendrocyte progenitor cells. J Neurosurg 99(2):344-350

23. Colin C et al (2006) In vitro identification and functional characterization of glial precursor cells in human gliomas. Neuropathol Appl Neurobiol 32(2):189-202

24. Colin C et al (2007) Relevance of combinatorial profiles of intermediate filaments and transcription factors for glioma histogenesis. Neuropathol Appl Neurobiol 33(4):431-439

25. Figarella-Branger D et al (1999) The PEN5 epitope identifies an oligodendrocyte precursor cell population and pilocytic astrocytomas. Am J Pathol 155(4):1261-1269

26. Ligon KL et al (2004) The oligodendroglial lineage marker OLIG2 is universally expressed in diffuse gliomas. J Neuropathol Exp Neurol 63(5):499-509

27. Potter N et al (2008) Genomic deletions correlate with underexpression of novel candidate genes at six loci in pediatric pilocytic astrocytoma. Neoplasia 10(8):757-772

28. Shoshan Y et al (1999) Expression of oligodendrocyte progenitor cell antigens by gliomas: implications for the histogenesis of brain tumors. Proc Natl Acad Sci USA 96(18):10361-10366

29. Takei $\mathrm{H}$ et al (2008) Expression of oligodendroglial differentiation markers in pilocytic astrocytomas identifies two clinical subsets and shows a significant correlation with proliferation 
index and progression free survival. J Neurooncol 86(2):183190

30. Paquin A et al (2005) CCAAT/enhancer-binding protein phosphorylation biases cortical precursors to generate neurons rather than astrocytes in vivo. J Neurosci 25(46):10747-10758

31. Carbonell WS, Mandell JW (2003) Transient neuronal but persistent astroglial activation of ERK/MAP kinase after focal brain injury in mice. J Neurotrauma 20(4):327-336

32. Fyffe-Maricich SL et al (2011) The ERK2 mitogen-activated protein kinase regulates the timing of oligodendrocyte differentiation. J Neurosci 31(3):843-850

33. Furnari FB et al (2007) Malignant astrocytic glioma: genetics, biology, and paths to treatment. Genes Dev 21(21):2683-2710

34. Ichimura $\mathrm{K}$ et al (2004) Molecular pathogenesis of astrocytic tumours. J Neurooncol 70(2):137-160

35. Jeuken $J$ et al (2007) RAS/RAF pathway activation in gliomas: the result of copy number gains rather than activating mutations. Acta Neuropathol 114(2):121-133

36. TCGA (2008) Comprehensive genomic characterization defines human glioblastoma genes and core pathways. Nature 455(7216): 1061-1068

37. Parsons DW et al (2008) An integrated genomic analysis of human glioblastoma multiforme. Science 321(5897):1807-1812

38. Huson SM et al (1989) A genetic study of von Recklinghausen neurofibromatosis in south east Wales. I. Prevalence, fitness, mutation rate, and effect of parental transmission on severity. J Med Genet 26(11):704-711

39. Takano $\mathrm{T}$ et al (1992) Genetics of neurofibromatosis 1 in Japan: mutation rate and paternal age effect. Hum Genet 89(3):281-286

40. DeClue JE et al (1992) Abnormal regulation of mammalian p21ras contributes to malignant tumor growth in von Recklinghausen (type 1) neurofibromatosis. Cell 69(2):265-273

41. Bennett MR et al (2003) Aberrant growth and differentiation of oligodendrocyte progenitors in neurofibromatosis type 1 mutants. J Neurosci 23(18):7207-7217

42. Lee JS et al (2010) Oligodendrocyte progenitor cell numbers and migration are regulated by the zebrafish orthologs of the NF1 tumor suppressor gene. Hum Mol Genet 19(23):4643-4653

43. Raff MC, Miller RH, Noble M (1983) A glial progenitor cell that develops in vitro into an astrocyte or an oligodendrocyte depending on culture medium. Nature 303(5916):390-396

44. Rodriguez EF et al (2011) PI3 K/AKT pathway alterations are associated with clinically aggressive and histologically anaplastic subsets of pilocytic astrocytoma. Acta Neuropathol 121(3):407-420

45. Listernick R, Charrow J, Gutmann DH (1999) Intracranial gliomas in neurofibromatosis type 1. Am J Med Genet 89(1):38-44

46. Rodriguez FJ et al (2008) Gliomas in neurofibromatosis type 1: a clinicopathologic study of 100 patients. J Neuropathol Exp Neurol 67(3):240-249

47. Lewis RA (1984) et al. von Recklinghausen neurofibromatosis. II. Incidence of optic gliomata. Ophthalmology 91(8):929-935

48. Gutmann DH et al (1997) The diagnostic evaluation and multidisciplinary management of neurofibromatosis 1 and neurofibromatosis 2. JAMA 278(1):51-57

49. Garner A, Klinworth G (1994) Tumours of the orbit, optic nerve and lacrimal sac. In: Garner A, Klintworth G (eds) Pathobiology of ocular disease: a dynamic approach, 2nd edn, vol. 2. Marcel Dekker, New York

50. Hernaiz Driever P et al (2010) Natural history and management of low-grade glioma in NF-1 children. J Neurooncol 100(2):199-207

51. Sharif S et al (2011) A molecular analysis of individuals with neurofibromatosis type 1 (NF1) and optic pathway gliomas (OPGs), and an assessment of genotype-phenotype correlations. J Med Genet 48(4):256-260
52. Fryssira $\mathrm{H}$ et al (2008) Tumor development in three patients with Noonan syndrome. Eur J Pediatr 167(9):1025-1031

53. Sanford RA et al (1999) A 16-year-old male with Noonan's syndrome develops progressive scoliosis and deteriorating gait. Pediatr Neurosurg 30(1):47-52

54. Schuettpelz LG et al (2009) Pilocytic astrocytoma in a child with Noonan syndrome. Pediatr Blood Cancer 53(6):1147-1149

55. Aoki Y et al (2008) The RAS/MAPK syndromes: novel roles of the RAS pathway in human genetic disorders. Hum Mutat 29(8):992-1006

56. Bar EE et al (2008) Frequent gains at chromosome 7q34 involving BRAF in pilocytic astrocytoma. J Neuropathol Exp Neurol 67(9):878-887

57. Deshmukh H et al (2008) High-resolution, dual-platform aCGH analysis reveals frequent HIPK2 amplification and increased expression in pilocytic astrocytomas. Oncogene 27(34):4745-4751

58. Pfister $\mathrm{S}$ et al. (2008) BRAF gene duplication constitutes a mechanism of MAPK pathway activation in low-grade astrocytomas. J Clin Invest 118:1739-1749

59. Jones DTW et al (2008) Tandem duplication producing a novel oncogenic BRAF fusion gene defines the majority of pilocytic astrocytomas. Cancer Res 68(21):8673-8677

60. Sievert AJ et al (2009) Duplication of 7q34 in pediatric lowgrade astrocytomas detected by high-density single-nucleotide polymorphism-based genotype arrays results in a novel BRAF fusion gene. Brain Pathol 19(3):449-458

61. Cin $\mathrm{H}$ et al (2011) Oncogenic FAM131B-BRAF fusion resulting from $7 \mathrm{q} 34$ deletion comprises an alternative mechanism of MAPK pathway activation in pilocytic astrocytoma. Acta Neuropathol 121(6):763-774

62. Forshew T et al (2009) Activation of the ERK/MAPK pathway: a signature genetic defect in posterior fossa pilocytic astrocytomas. J Pathol 218(2):172-181

63. Hasselblatt M et al. (2011) BRAF-KIAA1549 fusion transcripts are less frequent in pilocytic astrocytomas diagnosed in adults. Neuropathol Appl Neurobiol (in press)

64. Hawkins C et al. (2011) BRAF-KIAA1549 Fusion Predicts Better Clinical Outcome in Pediatric Low-Grade Astrocytoma. Clin Cancer Res (in press)

65. Jacob K et al (2009) Duplication of 7q34 is specific to juvenile pilocytic astrocytomas and a hallmark of cerebellar and optic pathway tumours. Br J Cancer 101(4):722-733

66. Korshunov A et al (2009) Combined molecular analysis of BRAF and IDH1 distinguishes pilocytic astrocytoma from diffuse astrocytoma. Acta Neuropathol 118(3):401-405

67. Lawson AR et al (2010) RAF gene fusions are specific to pilocytic astrocytoma in a broad paediatric brain tumour cohort. Acta Neuropathol 120(2):271-273

68. Schiffman JD et al (2010) Oncogenic BRAF mutation with CDKN2A inactivation is characteristic of a subset of pediatric malignant astrocytomas. Cancer Res 70(2):512-519

69. Yu J et al (2009) Alterations of BRAF and HIPK2 loci predominate in sporadic pilocytic astrocytoma. Neurology 73(19): 1526-1531

70. Ciampi R et al (2005) Oncogenic AKAP9-BRAF fusion is a novel mechanism of MAPK pathway activation in thyroid cancer. J Clin Invest 115(1):94-101

71. Dessars B et al (2007) Chromosomal translocations as a mechanism of BRAF activation in two cases of large congenital melanocytic nevi. J Invest Dermatol 127(6):1468-1470

72. Palanisamy $\mathrm{N}$ et al (2010) Rearrangements of the RAF kinase pathway in prostate cancer, gastric cancer and melanoma. Nat Med 16(7):793-798

73. Dougherty MJ et al. (2010) Activating mutations in BRAF characterize a spectrum of pediatric low-grade gliomas. Neuro Oncol 12:621-630 
74. Johnson MW et al (2010) Spectrum of pilomyxoid astrocytomas: intermediate pilomyxoid tumors. Am J Surg Pathol 34(12):1783-1791

75. Hastings PJ, Ira G, Lupski JR (2009) A microhomology-mediated break-induced replication model for the origin of human copy number variation. PLoS Genet 5(1):e1000327

76. Lawson AR et al (2011) RAF gene fusion breakpoints in pediatric brain tumors are characterized by significant enrichment of sequence microhomology. Genome Res 21(4):505-514

77. Davies $\mathrm{H}$ et al (2002) Mutations of the BRAF gene in human cancer. Nature 417(6892):949-954

78. Michaloglou C et al (2008) BRAF(E600) in benign and malignant human tumours. Oncogene 27(7):877-895

79. Wan PT et al (2004) Mechanism of activation of the RAF-ERK signaling pathway by oncogenic mutations of B-RAF. Cell 116(6):855-867

80. Eisenhardt AE et al. (2011) Functional characterization of a BRAF insertion mutant associated with pilocytic astrocytoma. Int J Cancer (in press)

81. Jones DTW et al (2009) Oncogenic RAF1 rearrangement and a novel BRAF mutation as alternatives to KIAA1549:BRAF fusion in activating the MAPK pathway in pilocytic astrocytoma. Oncogene 28(20):2119-2123

82. Schindler G et al (2011) Analysis of BRAF V600E mutation in 1, 320 nervous system tumors reveals high mutation frequencies in pleomorphic xanthoastrocytoma, ganglioglioma and extra-cerebellar pilocytic astrocytoma. Acta Neuropathol 121(3):397-405

83. Maltzman TH et al (1997) Ras oncogene mutations in childhood brain tumors. Cancer Epidemiol Biomarkers Prev 6(4):239-243

84. Janzarik WG et al (2007) Further evidence for a somatic KRAS mutation in a pilocytic astrocytoma. Neuropediatrics 38(2):6163

85. Sharma MK et al (2005) RAS pathway activation and an oncogenic RAS mutation in sporadic pilocytic astrocytoma. Neurology 65(8):1335-1336

86. Dasgupta B et al (2005) Glioma formation in neurofibromatosis 1 reflects preferential activation of K-RAS in astrocytes. Cancer Res 65(1):236-245

87. Cheng Y et al (2000) Pilocytic astrocytomas do not show most of the genetic changes commonly seen in diffuse astrocytomas. Histopathology 37(5):437-444

88. Duerr EM et al (1998) PTEN mutations in gliomas and glioneuronal tumors. Oncogene 16(17):2259-2264

89. Hayes VM et al (1999) High frequency of TP53 mutations in juvenile pilocytic astrocytomas indicates role of TP53 in the development of these tumors. Brain Pathol 9(3):463-467

90. Lang FF et al (1994) High frequency of p53 protein accumulation without $\mathrm{p} 53$ gene mutation in human juvenile pilocytic, low grade and anaplastic astrocytomas. Oncogene 9(3):949-954

91. Hudson TJ et al (2010) International network of cancer genome projects. Nature 464(7291):993-998

92. Jones DTW et al (2011) Adult grade II diffuse astrocytomas are genetically distinct from and more aggressive than their paediatric counterparts. Acta Neuropathol 121(6):753-761

93. Jacob K et al. (2011) Genetic aberrations leading to MAPK pathway activation mediate oncogene-induced senescence in sporadic pilocytic astrocytomas. Clin Cancer Res (in press)

94. Raabe EH et al (2011) BRAF activation induces transformation and then senescence in human neural stem cells: a pilocytic astrocytoma model. Clin Cancer Res 17(11):3590-3599

95. Serrano M et al (1997) Oncogenic ras provokes premature cell senescence associated with accumulation of p53 and p16INK4a. Cell 88(5):593-602

96. Courtois-Cox S et al (2006) A negative feedback signaling network underlies oncogene-induced senescence. Cancer Cell 10(6):459-472
97. Michaloglou C et al (2005) BRAFE600-associated senescencelike cell cycle arrest of human naevi. Nature 436(7051):720-724

98. Abel TW et al (2009) GFAP-Cre-mediated activation of oncogenic K-ras results in expansion of the subventricular zone and infiltrating glioma. Mol Cancer Res 7(5):645-653

99. de Vries NA et al (2010) Rapid and robust transgenic high-grade glioma mouse models for therapy intervention studies. Clin Cancer Res 16(13):3431-3441

100. Ding $\mathrm{H}$ et al (2001) Astrocyte-specific expression of activated p21-ras results in malignant astrocytoma formation in a transgenic mouse model of human gliomas. Cancer Res 61(9):38263836

101. Holland EC et al (2000) Combined activation of Ras and Akt in neural progenitors induces glioblastoma formation in mice. Nat Genet 25(1):55-57

102. Marumoto T et al (2009) Development of a novel mouse glioma model using lentiviral vectors. Nat Med 15(1):110-116

103. Uhrbom L et al (2002) Ink4a-Arf loss cooperates with KRas activation in astrocytes and neural progenitors to generate glioblastomas of various morphologies depending on activated Akt. Cancer Res 62(19):5551-5558

104. Uhrbom L et al (2005) Cell type-specific tumor suppression by Ink4a and Arf in Kras-induced mouse gliomagenesis. Cancer Res 65(6):2065-2069

105. Brannan CI et al (1994) Targeted disruption of the neurofibromatosis type-1 gene leads to developmental abnormalities in heart and various neural crest-derived tissues. Genes Dev 8(9):1019-1029

106. Jacks $\mathrm{T}$ et al (1994) Tumour predisposition in mice heterozygous for a targeted mutation in Nf1. Nat Genet 7(3):353-361

107. Bajenaru ML et al (2003) Optic nerve glioma in mice requires astrocyte Nf1 gene inactivation and Nf1 brain heterozygosity. Cancer Res 63(24):8573-8577

108. Yang FC et al (2008) Nf1-dependent tumors require a microenvironment containing $\mathrm{Nf} 1 \pm$ and c-kit-dependent bone marrow. Cell 135(3):437-448

109. Bajenaru ML et al (2002) Astrocyte-specific inactivation of the neurofibromatosis 1 gene (NF1) is insufficient for astrocytoma formation. Mol Cell Biol 22(14):5100-5113

110. Simmons GW et al (2011) Neurofibromatosis-1 heterozygosity increases microglia in a spatially and temporally restricted pattern relevant to mouse optic glioma formation and growth. J Neuropathol Exp Neurol 70(1):51-62

111. Warrington NM et al (2007) Spatiotemporal differences in CXCL12 expression and cyclic AMP underlie the unique pattern of optic glioma growth in neurofibromatosis type 1 . Cancer Res 67(18):8588-8595

112. Zhu $Y$ et al (2005) Inactivation of NF1 in CNS causes increased glial progenitor proliferation and optic glioma formation. Development 132(24):5577-5588

113. Kwon $\mathrm{CH}$ et al (2008) Pten haploinsufficiency accelerates formation of high-grade astrocytomas. Cancer Res 68(9):3286-3294

114. Zhu Y et al (2005) Early inactivation of p53 tumor suppressor gene cooperating with NF1 loss induces malignant astrocytoma. Cancer Cell 8(2):119-130

115. Lyustikman $\mathrm{Y}$ et al (2008) Constitutive activation of Raf-1 induces glioma formation in mice. Neoplasia 10(5):501-510

116. Robinson JP et al (2010) Activated BRAF induces gliomas in mice when combined with Ink4a/Arf loss or Akt activation. Oncogene 29(3):335-344

117. Robinson JP et al (2011) Activated MEK cooperates with Ink4a/ Arf loss or Akt activation to induce gliomas in vivo. Oncogene 30(11):1341-1350

118. Gronych $J$ et al (2011) An activated mutant BRAF kinase domain is sufficient to induce pilocytic astrocytoma in mice. J Clin Invest 121(4):1344-1348 
119. Gajjar A (1997) et al. Low-grade astrocytoma: a decade of experience at St. Jude Children's Research Hospital. J Clin Oncol 15(8):2792-2799

120. Gnekow A et al (2005) HIT-LGG 1996 - Success of a countrywide, comprehensive treatment strategy for children and adolescents with low grade glioma of all histologies and locations. Pediatr Blood Cancer 45(4):465

121. Wisoff $\mathrm{JH}$ et al. (2003) Impact of surgical resection on low grade gliomas of childhood: A report from the CCG9891/
POG9130 low grade astrocytoma study. Proc Am Soc Clin Oncol 22 Abstr. 401

122. Packer RJ et al (1997) Carboplatin and vincristine chemotherapy for children with newly diagnosed progressive low-grade gliomas. J Neurosurg 86(5):747-754

123. Perilongo G (2005) Considerations on the role of chemotherapy and modern radiotherapy in the treatment of childhood low grade glioma. J Neurooncol 75(3):301-307 